\section{Identificación y clasificación de las lesiones relacionadas con la dependencia: de la teoría a la práctica clínica}

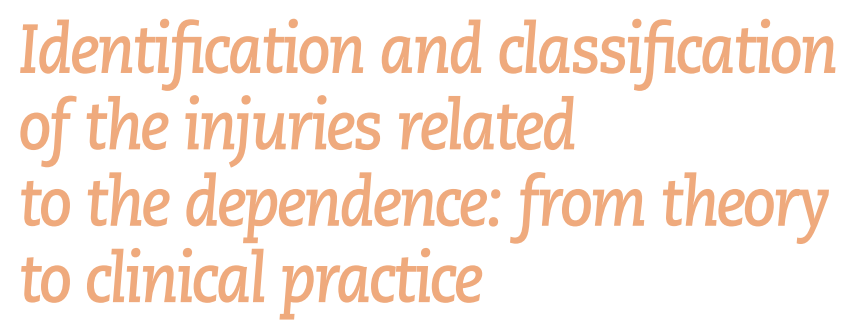

\author{
Alba Roca-Biosca' \\ Francisco Pedro García-Fernández ${ }^{2}$ \\ Sagrario Chacón-Garcés ${ }^{1}$ \\ Lourdes Rubio-Rico' ${ }^{1}$ \\ María Inmaculada de Molina-Fernández ${ }^{1}$ \\ Laura Anguera-Saperas ${ }^{3}$ \\ Natalia García-Grau ${ }^{4}$ \\ GemmaTuset-Garijo ${ }^{4}$ \\ María del Carmen Velasco Guillén ${ }^{4}$ \\ Encarna Colodrero-Díaz ${ }^{4}$ \\ 1. Departamento de Enfermería. Universidad Rovira i Virgili. Tarragona. \\ 2. Unidad de Estrategia de Cuidados. Complejo Hospitalario de Jaén. Comité Director \\ del GNEAUPP. \\ 3. Unidad de acogida prequirúrgica. Hospital Universitari Joan XXIII. Tarragona. \\ 4. Servicio de Medicina Intensiva. Hospital Universitari Joan XXIII. Tarragona.
}

Correspondencia:
Alba Roca Biosca
Avda.Catalunya 22D, 7. ${ }^{3}$. $^{\text {a }}$
43002 Tarragona
Tel.: 636130235
E-mail: alba.roca@urv.cat 
Presión y Heridas Crónicas (GNEAUPP) publicó el documento técnico n. ${ }^{\circ} \mathrm{II}^{4}$ que incorpora la posibilidad de que estas lesiones aparezcan también sobre tejidos blandos sometidos a presión externa por distintos materiales o dispositivos clínicos usados con fines diagnósticos o terapéuticos (p. ej., sonda nasogástrica, tubo endotraqueal, catéter urinario, etc.).

El único modelo sobre el mecanismo de producción de las UPP que se ha publicado en la literatura internacional es el realizado en 1987 por las Dras. Barbara Braden y Nancy Bergstrom5 . Aunque dicho modelo identifica diversos factores implicados en el desarrollo de las UPP, hasta el momento, este ha sido el único término empleado para designar tanto aquellas lesiones cuyo mecanismo causal es la presión, como aquellas que responden a etiologías distintas. Las investigaciones llevadas a cabo en los últimos años sobre el tema llevaron a García-Fernández y cols. ${ }^{6}$ al desarrollo de una nueva teoría enfermera de grado medio que incluía la construcción de un modelo que explica no solo el mecanismo de producción de las UPP, sino el de hasta siete tipos de lesiones distintas a las que denominaron lesiones relacionadas con la dependencia (LRD). Este término engloba las lesiones por presión-cizalla, las lesiones por roce-fricción (LF), las cutáneas asociadas a la humedad (LESCAH) así como otros tres tipos de lesiones que resultan de su combinación (presión-humedad, presión-fricción y humedad-fricción). Por último, también se establece un tipo de lesión que se denomina multifactorial. Al hablar de LRD, los autores hacen referencia a la dependencia como único elemento en común que comparten todas ellas.

El término dependencia es inherente al paciente crítico. Y es este alto grado de dependencia el que lo convierte en vulnerable a la aparición de LRD. El grado de dependencia de los pacientes como elemento favorecedor de la aparición de lesiones ha sido reconocido recientemente por Balzer y cols. ${ }^{7}$ en una investigación cualicuantitativa que evalúa qué características de los pacientes tienen en cuenta las enfermeras para valorar el riesgo de desarrollar UPP sin el empleo de una escala.

El presente estudio pretende ser una investigación aplicada a la práctica clínica de este nuevo modelo teórico, que a su vez conducirá en un futuro próximo a diferentes planteamientos tanto preventivos como terapéuticos. Tiene como objetivo clasificar-categorizar las LRD en función de la teoría de rango medio desarrollada por García-Fernández y cols.

\section{У MATERIAL Y MÉTODO}

Tipo de estudio: estudio descriptivo, observacional y longitudinal, realizado en la UCI polivalente de 14 camas del Hospital Universitari Joan XXIII de Tarragona.

Periodo de estudio: se inició el 13 de enero de 2014 y finalizó el 30 de junio del mismo año.

Criterios de inclusión, exclusión y salida de estudio: se incluyeron todos los pacientes mayores de edad que no presentaban LRD en el momento del ingreso. Se excluyeron, a priori, aquellos cuyo diagnóstico médico al ingreso fue de muerte encefálica. Los criterios de exclusión aplicados a posteriori fueron: menos de 2 días de estancia y/o más de 2 días consecutivos sin recoger datos. Los pacientes permanecieron en el estudio hasta que desarrollaron una LRD de categoría I o superior, fallecieron, fueron dados de alta o su estancia en la unidad superó los 14 días.

Tamaño de la muestra y tipo de muestreo: se trata de una muestra de tipo conceptual, ya que incluye a toda la población del periodo de estudio. El muestreo es de conveniencias o accidental, que incluye a todos los pacientes ingresados entre las fechas de estudio.

Métodos para la obtención de los datos: cada paciente fue evaluado diariamente por una enfermera del grupo investigador hasta la salida de estudio. Las enfermeras del grupo investigador participaron en reunio- nes de equipo con el fin de homogeneizar los criterios de clasificación y categorización de las LRD. Para la recogida diaria de datos se diseñó una hoja de elaboración propia que incluía cuestiones referentes a datos sociodemográficos y clínicos del paciente en el momento del ingreso. En el caso de aparición de una LRD se fotografiaba y se anotaban los datos relacionados con ella (tipo de lesión, categoría y localización), así como otra información orientada a una correcta clasificación de la lesión. La clasificación se llevó a cabo basándose en lo descrito en el Documento Técnico del GNEAUPP n. ${ }^{\circ}$ II de Clasificación-Categorización, elaborado con el fin de ayudar a la comprensión de este nuevo modelo ${ }^{4}$.

La cámara que se utilizó para la toma de fotografías poseía una resolución de 12 megapíxeles, lo cual permitió una correcta visualización de la lesión sin distorsión óptica. Se procuró que las fotografías fueran tomadas en posición perpendicular (angular de $90^{\circ}$ ) y con la función de flash activa. Las imágenes de las lesiones se almacenaron en un archivo fotográfico y fueron identificadas con el código de paciente. Aunque el tipo de LRD se determinaba en el momento de su aparición, semanalmente el grupo investigador se reunía para confirmar la valoración inicial y consensuar aquellos casos que generaban controversia mediante la revisión de las imágenes de las lesiones y la información anotada en la hoja de recogida de datos.

De manera protocolizada, cada paciente fue valorado según la escala EMINA $^{8}$ por la enfermera asistencial y en función de esta valoración se aplicaron las medidas preventivas necesarias.

Análisis de los datos: se ha realizado un análisis de tipo secuencial, primero univariante (de tipo descriptivo) donde las variables cuantitativas se presentan como media y desviación estándar, y las variables cualitativas como frecuencias absolutas y porcentajes. Posteriormente se ha realizado un análisis bivariante para comparar las características de los pacientes con y sin LRD mediante la $t$ de Student (tras comparar normalidad mediante la prueba de K-S) para las variables cuantitativas y la $\chi^{2}$ para las cualitativas. En todos los casos se consideró significativa una $p \leq 0,05$.

Aspectos éticos y legales de los sujetos participantes: el proyecto cuenta con la aprobación del Comité Ético de Investigaciones Clínicas (CEIC) del Hospital Universitari Joan XXIII de Tarragona (CEIC 02/2014). Los datos fueron almacenados en una base del programa SPSS versión 19.0 que cumplía con los criterios de privacidad establecidos por la Ley Orgánica 15/1999 de 13 de diciembre y de Protección de Datos de Carácter Personal.

\section{$\searrow$ RESULTADOS}

La población accesible fueron 178 pacientes, de los cuales se incluyeron en el estudio un total de 136. La figura 1 representa el diagrama de flujo de la muestra y se explican los motivos de exclusión del estudio: 20 lo fueron, a priori (fundamentalmente por presentar UPP al ingreso), y 22 a posteriori (principalmente por estancia inferior a $48 \mathrm{~h}$ ).

Del total de pacientes estudiados, 38 desarrollaron LRD. Esto supone una incidencia del 27,9\%. Los datos referentes a la comparación de las variables estudiadas entre ambas poblaciones se muestran en la tabla 1. El perfil del paciente con LRD responde a un adulto mayor, varón y obeso, con un diagnóstico de tipo médico y un riesgo alto de desarrollar UPP según la escala EMINA. No se hallaron diferencias estadísticamente significativas entre los pacientes que desarrollaron LRD y aquellos que no lo hicieron salvo para la variable EMINA del primer día $(p=$ $0,035)$. Los pacientes que desarrollaron LRD puntuaban más alto en la valoración del riesgo medido con la escala EMINA que el resto de pacientes incluidos en el estudio. 


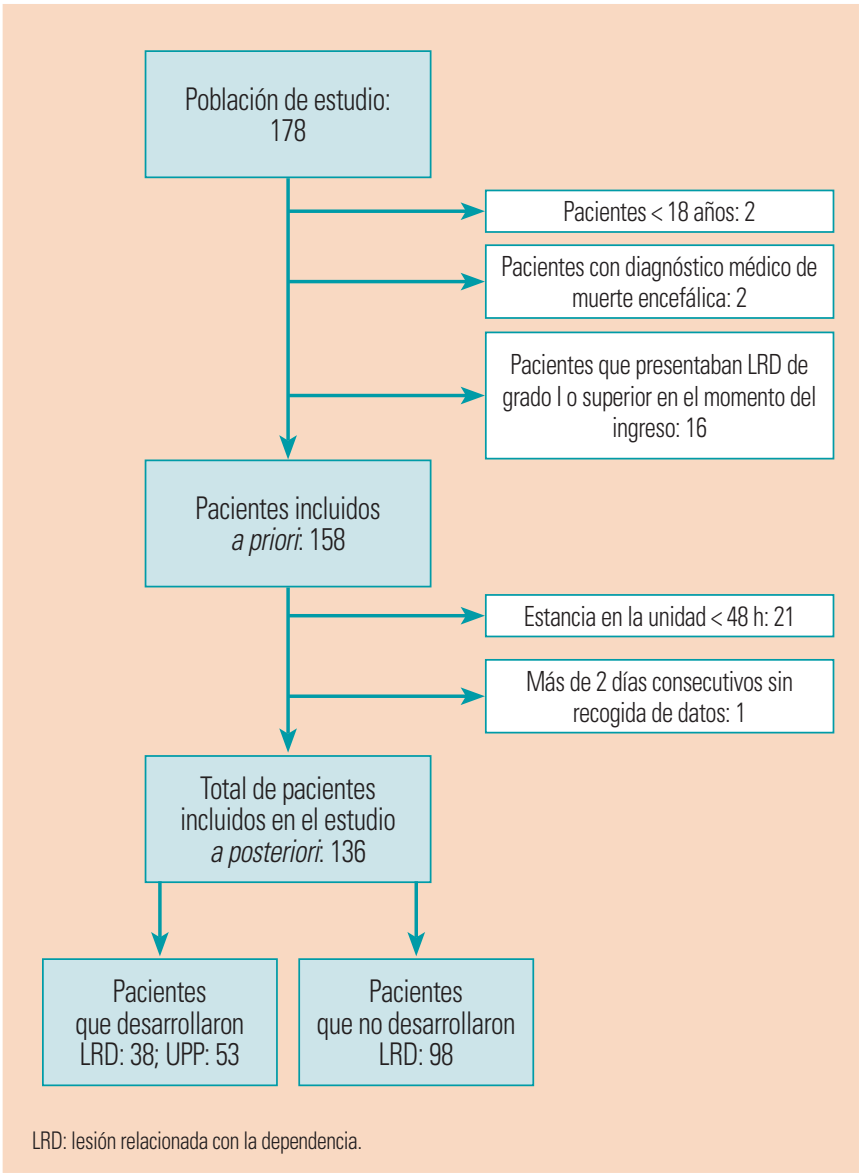

La distribución de las LRD según su mecanismo causal así como su categorización se muestra en la tabla 2. En la tabla solo se han incluido aquellas LRD y/o categorías de lesión que tenían representación. Es interesante destacar que el $46,4 \%$ de las LRD se catalogaron como UPP y que esta fue la categoría mayoritaria, seguida de las LF y LESCAH, correlativamente.

La localización más frecuente de las LRD fue en talón, nariz y nalgas por igual, seguido de orejas y sacro. Estos resultados se muestran de manera detallada en la tabla 3 .

Es importante resaltar que del total de lesiones, $14(36,8 \%)$ se relacionaron con la presencia de dispositivos terapéuticos (sonda nasogástrica y tubo endotraqueal).

Como soporte a la categorización detallada en las tablas previas se ha elaborado una figura (fig. 2) en la que se muestra una fotografía representativa de cada uno de los tipos de lesión identificados.

\section{У DISCUSIÓN}

Las lesiones detectadas en la práctica clínica se han podido clasificar según el modelo de García-Fernández y cols. ${ }^{6}$, lo que ha permitido diferenciar entre UPP y otro tipo de lesiones en pacientes con alto grado de dependencia. Nuestros resultados apoyan el cambio de paradigma propuesto por estos autores ${ }^{6}$. Agrupar bajo una única denominación (UPP) a lesiones cuyo origen es en ocasiones muy distinto a la presión entorpecía la labor preventiva orientada a contrarrestar los efectos de los diferentes mecanismos causales. Los resultados de este estudio avalan estas conclusiones, puesto que la presión como factor etiológico único solo está presente en el $48 \%$ de las lesiones.

Las características de los pacientes que desarrollaron LRD en cuando a media de edad y sexo coinciden con la literatura especializada publi-

Figura 1. Diseño del estudio.

Tabla 1. Comparación de variables entre población estudiada y pacientes que desarrollaron lesiones relacionadas con la dependencia

\begin{tabular}{|c|c|c|c|c|}
\hline \multirow{2}{*}{\multicolumn{2}{|c|}{ Media de edad }} & $\begin{array}{l}\text { Pacientes sin lesión relacionada } \\
\text { con la dependencia } \\
\text { ( } \mathrm{n}=98)\end{array}$ & $\begin{array}{l}\text { Pacientes con lesión relacionada } \\
\text { con la dependencia } \\
(\mathbf{n}=38)\end{array}$ & $p$ \\
\hline & & $\bar{X}=60,6 ; \mathrm{DE}: 14,9$ & $\bar{X}=61,2 ; \mathrm{DE}: 13$ & 0,8 \\
\hline \multicolumn{2}{|c|}{ Índice de masa corporal } & $\bar{X}=26,2 ; \mathrm{DE}: 4$ & $\bar{X}=33 ; \mathrm{DE}: 31,9$ & 0,2 \\
\hline \multicolumn{2}{|c|}{ Días de ingreso previo } & $\bar{X}=3,6 ; \mathrm{DE}: 8$ & $\bar{X}=4,3 ; D E: 13,2$ & 0,7 \\
\hline \multicolumn{2}{|c|}{ Apache II al ingreso } & $\bar{X}=17,7 ; \mathrm{DE}: 7$ & $\bar{X}=17,3 ; D E: 7,6$ & 0,8 \\
\hline \multicolumn{2}{|c|}{ Media EMINA del primer día } & $\bar{X}=10,3 ; D E: 2,1$ & $\bar{X}=11 ; \mathrm{DE}: 1,6$ & 0,03 \\
\hline \multicolumn{2}{|l|}{ Hombres } & $66(67,3 \%)$ & $29(76,3 \%)$ & 0,3 \\
\hline \multirow{4}{*}{ Grupo diagnóstico } & Médico & $49(50 \%)$ & $20(52,6 \%)$ & 0,9 \\
\hline & Quirúrgico & $32(32,7 \%)$ & $11(28,9 \%)$ & 0,8 \\
\hline & Traumático & $8(8,2 \%)$ & $2(5,3 \%)$ & 0,8 \\
\hline & Neurocrítico & $9(9,2 \%)$ & $5(13,2 \%)$ & 0,7 \\
\hline \multirow{4}{*}{ Procedencia } & Urgencias & $34(34,7 \%)$ & $12(31,6 \%)$ & 0,9 \\
\hline & Quirófano & $28(28,6 \%)$ & $9(23,7 \%)$ & 0,7 \\
\hline & Traslado & $22(22,4 \%)$ & $9(23,7 \%)$ & 0,9 \\
\hline & UCIM & $14(14,3 \%)$ & $8(21,1 \%)$ & 0,4 \\
\hline
\end{tabular}


Tabla 2. Clasificación-categorización de las lesiones relacionadas con la dependencia

\begin{tabular}{|c|c|c|c|c|}
\hline Tाpo de lesión & $n=38(\%)$ & Categoría & n & $\%$ \\
\hline \multirow{6}{*}{ Úlceras por presión } & \multirow{6}{*}{$14(36,8 \%)$} & Cat. I & 3 & 7,8 \\
\hline & & \multirow{4}{*}{ Cat. II } & 7 & 18,4 \\
\hline & & & 1 & 2,6 \\
\hline & & & 1 & 2,6 \\
\hline & & & 1 & 2,6 \\
\hline & & Cat. III & 1 & 2,6 \\
\hline \multirow{3}{*}{ Úlceras por presión/cizalla } & \multirow{3}{*}{$4(10,5 \%)$} & \multirow{2}{*}{ Cat. I } & 2 & 5,2 \\
\hline & & & 1 & 2,6 \\
\hline & & Cat. II & 1 & 2,6 \\
\hline \multirow{5}{*}{$\begin{array}{l}\text { Lesiones asociadas } \\
\text { a la humedad }\end{array}$} & \multirow{5}{*}{$5(13,2 \%)$} & 1B & 1 & 2,6 \\
\hline & & \multirow{2}{*}{$2 \mathrm{~A}$} & 1 & 2,6 \\
\hline & & & 1 & 2,6 \\
\hline & & \multirow{2}{*}{$2 \mathrm{~B}$} & 1 & 2,6 \\
\hline & & & 1 & 2,6 \\
\hline \multirow{5}{*}{ Lesiones por roce-fricción } & \multirow{5}{*}{$6(15,8 \%)$} & \multirow{2}{*}{ Cat. II } & 2 & 5,2 \\
\hline & & & 1 & 2,6 \\
\hline & & \multirow{3}{*}{ Cat. III } & 1 & 2,6 \\
\hline & & & 1 & 2,6 \\
\hline & & & 1 & 2,6 \\
\hline \multirow{2}{*}{$\begin{array}{l}\text { Lesiones combinadas } \\
\text { presión-humedad }\end{array}$} & \multirow{2}{*}{$4(10,5 \%)$} & Cat. II-2A & 2 & 5,2 \\
\hline & & Cat. II-2B & 2 & 5,2 \\
\hline \multirow{2}{*}{$\begin{array}{l}\text { Lesiones combinadas presión } \\
\text { fricción }\end{array}$} & \multirow{2}{*}{$3(7,8 \%)$} & $\|-\|$ & 2 & 5,2 \\
\hline & & H & 1 & 2,6 \\
\hline \multirow{2}{*}{$\begin{array}{l}\text { Lesiones combinadas fricción } \\
\text { humedad }\end{array}$} & \multirow{2}{*}{$2(5,3 \%)$} & $\| I \mid-2 A$ & 1 & 2,6 \\
\hline & & $\mathrm{I}-2 \mathrm{~A}$ & 1 & 2,6 \\
\hline
\end{tabular}

Tabla 3. Localización y categorización de las lesiones relacionadas con la dependencia

\begin{tabular}{|c|c|c|c|c|}
\hline Localización & $n=38(\%)$ & Тро & Categoría & $n(\%)$ \\
\hline \multirow{3}{*}{ Talón } & \multirow{3}{*}{$7(18,4)$} & UPP & Cat. I & $3(7,8)$ \\
\hline & & Presión-cizalla & Cat. I & $2(5,2)$ \\
\hline & & Roce-fricción & Cat. II & $2(5,2)$ \\
\hline Nariz & $7(18,4)$ & UPP & Cat. II & $7(18,4)$ \\
\hline \multirow{7}{*}{ Nalgas } & \multirow{7}{*}{$7(18,4)$} & \multirow{3}{*}{ LESCAH } & Cat. 1B & $1(2,6)$ \\
\hline & & & Cat. 2A & $1(2,6)$ \\
\hline & & & Cat. 2B & $1(2,6)$ \\
\hline & & Roce-fricción & Cat. III & $1(2,6)$ \\
\hline & & Combinadas presión-fricción & Cat. I-I & $1(2,6)$ \\
\hline & & $\begin{array}{c}\text { Combinadas fricción- } \\
\text { humedad }\end{array}$ & $\begin{array}{l}\text { Cat. } \\
\text { III-2A }\end{array}$ & $1(2,6)$ \\
\hline & & $\begin{array}{l}\text { Combinadas fricción- } \\
\text { humedad }\end{array}$ & Cat. I-2A & $1(2,6)$ \\
\hline \multirow{3}{*}{ Oreja } & \multirow{3}{*}{$5(13,2)$} & UPP & Cat. II & $1(2,6)$ \\
\hline & & $\begin{array}{c}\text { Combinadas presión- } \\
\text { humedad }\end{array}$ & Cat. II-2A & $2(5,2)$ \\
\hline & & Combinadas presión-fricción & Cat. II-\| & $2(5,2)$ \\
\hline \multirow{3}{*}{ Sacro } & \multirow{3}{*}{$3(7,9)$} & UPP & Cat. II & $1(2,6)$ \\
\hline & & \multirow{2}{*}{ Presión-cizalla } & Cat. I & $1(2,6)$ \\
\hline & & & Cat. II & $1(2,6)$ \\
\hline \multirow{2}{*}{ Muslo } & \multirow{2}{*}{$2(5,3)$} & \multirow{2}{*}{ Roce-fricción } & Cat. II & $1(2,6)$ \\
\hline & & & Cat. III & $1(2,6)$ \\
\hline \multirow{2}{*}{ Boca } & \multirow{2}{*}{$2(5,3)$} & \multirow{2}{*}{$\begin{array}{l}\text { Combinadas presión- } \\
\text { humedad }\end{array}$} & Cat. II-2A & $1(2,6)$ \\
\hline & & & Cat. II-2B & $1(2,6)$ \\
\hline Maléolo & $1(2,6)$ & UPP & Cat. II & $1(2,6)$ \\
\hline Codo & $1(2,6)$ & UPP & Cat. III & $1(2,6)$ \\
\hline Omóplato & $1(2,6)$ & Roce-fricción & Cat. III & $1(2,6)$ \\
\hline $\begin{array}{l}\text { Pliegues } \\
\text { abdominales }\end{array}$ & $1(2,6)$ & LESCAH & Cat. 2B & $1(2,6)$ \\
\hline Genitales & $1(2,6)$ & LESCAH & Cat. 2A & $1(2,6)$ \\
\hline
\end{tabular}

LESCAH: lesiones cutáneas asociadas a la humedad; UPP: úlceras por presión.

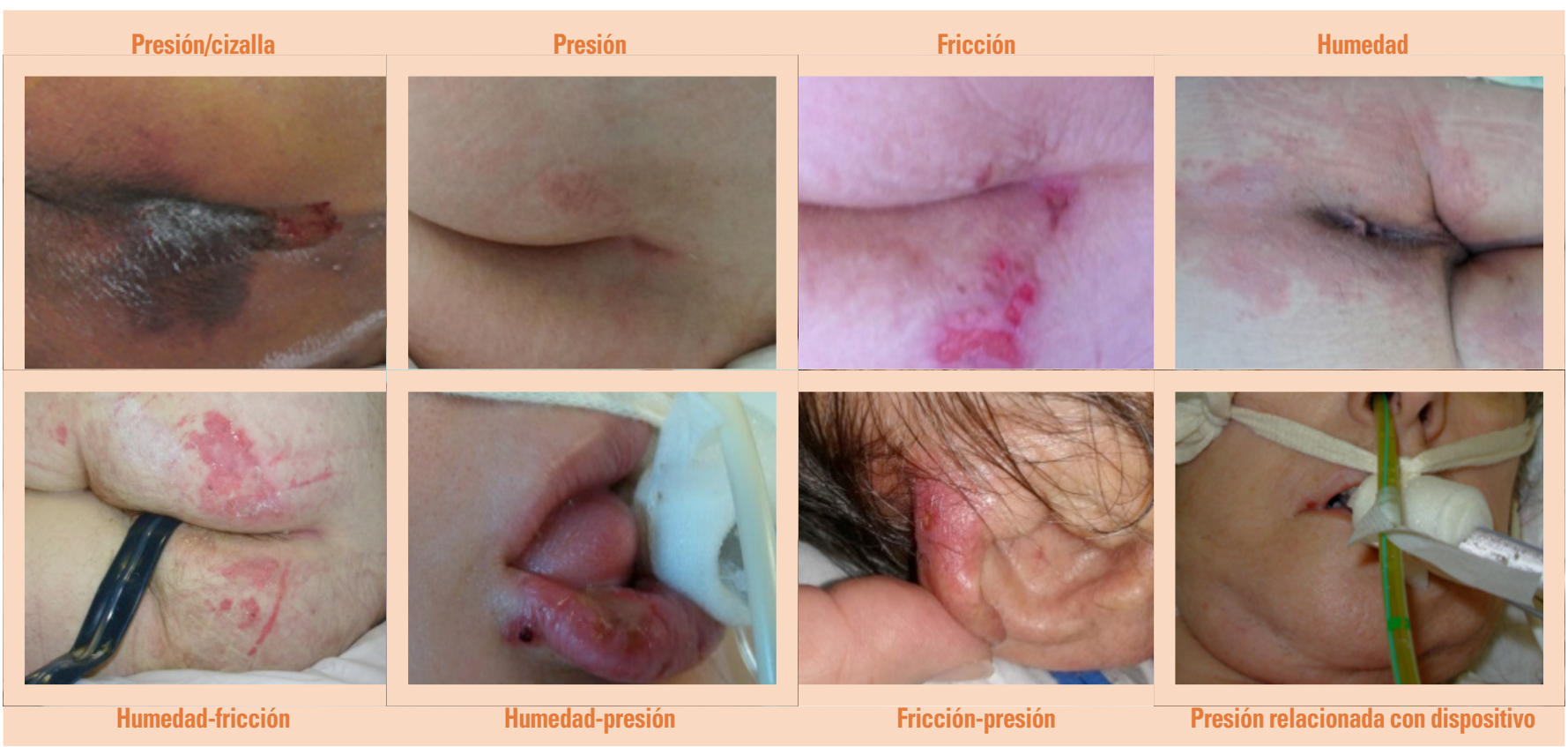

Figura 1. Fotografía representativa de cada uno de los tipos de lesión identificados. 
cada al respecto ${ }^{2,9,10}$. En referencia a la localización, aunque la mayoría de investigaciones sitúan al sacro como primera zona afectada ${ }^{9,11}$, en nuestro caso el porcentaje más elevado de lesiones se desarrolló en talón, nariz y nalgas por igual.

Las LESCAH y las LF de categoría I han pasado desapercibidas, se han diagnosticado directamente en categorías superiores. Algunos estudios publicados ${ }^{12-14}$ informan sobre la falta de habilidad de los profesionales de enfermería para identificar y diferenciar las lesiones por humedad del resto de lesiones. A menudo, en la práctica clínica la humedad se relaciona solo con las lesiones producidas por la orina y las heces dejando al margen que otros fluidos como el sudor, el exudado de la herida, el moco o la saliva también pueden intervenir directamente en su aparición ${ }^{4}$. En el presente estudio, las $\mathrm{LESCAH}^{15}$ se han identificado también en áreas al margen de glúteos o genitales, tales como la boca o los pliegues cutáneos (estas últimas conocidas como dermatitis intertriginosa o dermatitis por transpiración) y no acostumbran a ser catalogadas como tales por desconocimiento de los profesionales, a pesar de contar con mecanismos de prevención propios ${ }^{16}$.

Por lo que respecta a las LF, es comprensible que resulten difíciles de catalogar en fases iniciales, puesto que fue García-Fernández ${ }^{17}$ en el año 2011 quien les dio por primera vez entidad propia y pasó a considerarlas como independientes de la presión, tal y como venían agrupándose hasta el momento ${ }^{18}$. Más tarde otros autores han seguido aportando datos sobre características diferenciales que ayudan a discernir entre los diferentes tipos de lesiones ${ }^{19}$. La hoja de recogida de datos elaborada para este estudio basándose en estas aportaciones podría ser útil en la práctica clínica e investigadora, puesto que incorpora elementos para identificar/catalogar las diferentes LRD.

El 36,8\% de las LRD identificadas estaban relacionadas con el uso de dispositivos médicos y se localizaron en la nariz, orejas y boca. En el año 2010 Black y cols. ${ }^{20}$ llevaron a cabo un estudio con una amplia muestra de 2500 pacientes, del que se desprende que un tercio de las lesiones que se identificaron estaban relacionadas con dispositivos médicos. En su caso, la localización más frecuente fue las orejas. Dado que la existencia de protocolos de prevención de UPP es un hecho en la práctica clínica, el alto porcentaje de este tipo de lesiones se podría achacar, en parte, a su incumplimiento. Aunque, tal como proponen Patón y cols. ${ }^{21}$, creemos que la prevención de las LRD debería abordarse desde una perspectiva integral, como un ciclo de mejora continua que implique a todos los elementos del proceso asistencial y no únicamente a las enfermeras.

Como fortaleza del estudio cabe destacar el valor ańadido de la fotografía de las lesiones en la metodología utilizada. El análisis de las imágenes a posteriori permitió una clasificación más certera de las mismas. Por el contrario, se debe señalar la escasa representación en alguno de los grupos y categorías como posible limitación del presente estudio.

En esta investigación se ha utilizado la escala EMINA $^{8}$ para valorar el riesgo de desarrollar LRD. Se halló relación estadísticamente significativa entre la aparición de LRD y puntuaciones más altas en la valoración EMINA del primer día. A esta conclusión llegaron ya los mismos autores en un estudio llevado a cabo con anterioridad ${ }^{10}$. Este es un hecho importante y demuestra que la detección del riesgo debe considerarse parte fundamental del proceso asistencial. EMINA posee una buena capacidad predictiva ${ }^{22}$, pero no ha sido validada en un contexto de pacientes críticos. En este ámbito, la escala Braden ${ }^{5}$ es la más testada ${ }^{22-24}$. Por otra parte, Lahmann y cols. ${ }^{25}$ afirman que algunas subescalas de la escala Braden podrían estar relacionadas con la aparición de un determinado tipo y categoría de lesión. Ante esta afirmación cabe preguntarse por la posible relación entre las subescalas y las diferentes LRD establecidas en el nuevo modelo teórico de García-Fernández y cols. ${ }^{6}$. Dada la alta incidencia de LRD detectadas en este estudio, creemos que el contexto asistencial de UCI es idóneo para futuras investigaciones en esta línea.

\section{Conflicto de intereses}

Los autores declaran no presentar ningún conflicto de intereses. Uno de los autores es el creador del modelo teórico que se contrasta en este artículo

\section{У BIBLIOGRAFÍA}

1. Soldevilla Agreda JJ, Torra i Bou JE, Rueda López J, Martínez Cuervo F Verdú Soriano J, Mayán Santos JM, et al. $2{ }^{\circ}$ Estudio Nacional de Prevalencia de Úlceras por Presión en España, 2005. Epidemiología y variables definitorias de las lesiones y pacientes. Gerokomos. 2006:17:154-72.

2. Soldevilla Agreda JJ, Torra i Bou J-E, Verdú Soriano J, López Casanova P. 3.er Estudio Nacional de Prevalencia en España, 2009 Epidemiología y variables definitorias de las lesiones y pacientes. Gerokomos. 2011:22(2):77-90.

3. European Pressure Ulcer Advisory Panel and National Pressure Ulcer Advisory Panel. Prevention and treatment of pressure ulcers: quick reference guide. Washington DC: National Pressure Ulcer Advisory Panel; 2009.

4. García-Fernández FP, Soldevilla Ágreda JJ, Pancorbo-Hidalgo PL, Verdú Soriano J, López-Casanova, P, Rodríguez-Palma M. Clasificación-categorización de las lesiones relacionadas con la dependencia. Serie Documentos Técnicos GNEAUPP n. ${ }^{0}$ II. Grupo Nacional para el Estudio y Asesoramiento en Úlceras por Presión y Heridas Crónicas. Logroño, 2014. Disponible en: http://gneau$\mathrm{pp}$.info/clasificacion-categorizacion-de-las-lesiones-relacionadas-con-la-dependencia-2/

5. Braden B, Bergstrom N. A conceptual schema for the study of the etiology of pressure sores. Rehabil Nurs.1987;12:8-12.

6. Garcia-Fernandez FP, Agreda JJ, Verdu J, Pancorbo-Hidalgo PL. A new theoretical model for the development of pressure cers and other dependence-related lesions. J Nurs Scholarsh. 2014;46(1):28-38

7. Balzer K, Kremer L, Junghans A, Halfens RJG, Dassen T, Kottner J. What patient characteristics guide nurses' clinical judgement on pressure ulcer risk? A mixed methods study. Int J Nurs Stud. 2014:51(5):703-16.
8. Fuentelsaz Gallego C. Validación de la escala EMINA ${ }^{\odot}$ : un instrumento de valoración del riesgo de desarrollar úlceras por presión en pacientes hospitalizados. Enferm Clín. 2001;11(3):97-103.

9. Labiano-Turrillas J, Larrea-Leoz B, Vázquez-Calatayud M, Juandeaburre-Pedroarena B, Irruarizaga-Sagredo A, Morillo-Cabezas E. Estudio de prevalencia de úlceras por presión en la Clínica Universidad de Navarra. Gerokomos. 2013;24(4):184-8.

10. Roca-Biosca A, Velasco-Guillén MC, Rubio-Rico L, García-Grau N Anguera-Saperas L. Úlceras por presión en el enfermo crítico: detección de factores de riesgo. Enferm Intensiva. 2012;23:155-63.

11. Vanderwee K, Clark M, Dealey C, Gunningberg L, Defloor T. Pressure ulcer prevalence in Europe: a pilot study. J Eval Clin Pract. 2007;13:227-35

12. Segovia-Gómez T, Bermejo Martínez M, García-Alamino JM. Úlceras por humedad: conocerlas mejor para poder prevenirlas. Gerokomos. 2012;23(3):137-40.

13. Beeckman D, Schoonhoven L, Fletcher J, Furtado K, Gunningberg $\mathrm{L}$, Heyman $\mathrm{H}$, et al. EPUAP classification system for pressure ulcers: European reliability study. J Adv Nurs. 2007:60:682-91.

14. Mahoney M, Rozenboom B, Doughty D, Smith H. Issues related to accurate classification of buttocks wounds. J Wound Ostomy Continence Nurs. 2011:38:635-42.

15. Torra i Bou JE, Rodríguez Palma M, Soldevilla Agreda JJ, García-Fernández FP, Sarabia R, Zabala Blanco J, et al. Redefinición del concepto y del abordaje de las lesiones por humedad. Una propuesta conceptual y metodológica para mejorar el cuidado de las lesiones cutáneas asociadas a la humedad (LESCAH). Gerokomos. 2013;24(2):90-4.

16. Black JM, Gray M, Bliss DZ, Kennedy-Evans KL, Logan S, Baharestani MM, et al. MASD Part 2: Incontinence-Associated Dermatitis and Intertriginous Dermatitis: a consensus. J Wound Ostomy Continence Nurs. 2011;38(4):359-70; quiz 71-2.
17. Garcia-Fernandez FP. Escalas de valoración del riesgo de desarrollar úlceras por presión: Una revisión con meta-análisis [tesis doctoral]. Jaén (España): Universidad de Jaén; 2011.

18. Hanson D, Langemo DK, Anderson J, Thompson P, Hunter S. Friction and shear considerations in pressure ulcer development. Adv Skin Would Care. 2010;23(1):21-4.

19. Llatas FP, Pujalte BF, Areosa LA, Prieto JR. Diferenciación de las úlceras en pacientes encamados y con enfermedades crónicas. Influencia de la humedad, fricción, cizalla y presión. Enfermería Dermatológica. 2013;7(18):14-25.

20. Black JM, Cuddigan JE, Walko MA, Didier LA, Lander MJ, Kelpe MR. Medical device related pressure ulcers in hospitalized patients. Int Wound J. 2010;7:358-65

21. Patón Villar F, Lorente Granados G, Fernández-Lasquetty Blanc B, Hernández Martínez A, Escot Higueras S, Quero Palomino $\mathrm{M}$, et al. Plan de mejora continua en prevención-tratamiento de úlceras por presión según el ciclo de Deming. Gerokomos. 2013;24(3):125-31.

22. Garcia-Fernandez FP, Pancorbo-Hidalgo PL, Agreda JJ. Predictive capacity of risk assessment scales and clinical judgment for pressure ulcers: a meta-analysis. J Wound Ostomy Continence Nurs 2014;41(1):24-34.

23. Kelechi T, Arndt J, Dove A. Review of pressure ulcer risk assessment scales. J Wound Ostomy Continence Nurs. 2013;40(3):232-6.

24. Šáteková L, Žiakovák. Validity of pressure ulcer risk assessment scales: review. Cent Eur Nurs Midw. 2014;5(2)85-92.

25. Lahmann NA, Kotter J. Relation between pressure, friction and pressure ulcer categories: A secondary data analysis of hospital patients using CHAID methods. Int J Nurs Stud. 2011:48(12):1487-94. 